\title{
Transverse loading of multicore fibre gratings
}

\author{
M Silva-Lopez ${ }^{\mathrm{a}}$, D Zhao ${ }^{\mathrm{b}}, \mathrm{C} \mathrm{Li}^{\mathrm{a}}$, W N MacPherson ${ }^{\mathrm{a}}{ }^{*}, \mathrm{G} \mathrm{M} \mathrm{H}$ Flockhart $^{\mathrm{aql}}$, A J Moore ${ }^{\mathrm{a}}$, \\ J S Barton ${ }^{\mathrm{a}}$, J D C Jones ${ }^{\mathrm{a}}$, L Zhang ${ }^{\mathrm{b}}$, I Bennion ${ }^{\mathrm{b}}$ \\ ${ }^{a}$ School of Engineering and Physical Sciences, Heriot-Watt University, Edinburgh EH14 4AS, UK; \\ ${ }^{\mathrm{b}}$ Photonics Research Group, Electronic Engineering and Computer Science Division, Aston \\ University, Birmingham B4 7ET, UK
}

\begin{abstract}
We report experimental measurements of the reflection spectra of Bragg gratings inscribed in 4-core fibres under transverse loading. Broadening and splitting of the Bragg peaks from each core are observed as a function of load and fibre orientation.
\end{abstract}

Keywords: optical fibre sensors, multicore fibre, transverse stress, fibre Bragg grating

\section{INTRODUCTION}

The stresses induced in optical fibres by transverse loading were originally investigated in order to assess the potential for controllable coupling of light into or out of conventional fibre [1] and stress effects on highly birefringent fibres [2]. More recently there has been interest in using fibre Bragg gratings (FBGs) as transverse load sensors for engineering applications, opening up the possibility of three-axis load measurement by a single sensor. The birefringence induced by transverse stress in the fibre core produces a splitting of the FBG spectrum which can be modelled successfully by coupled mode theory [3]. In particular, FBGs written in highly birefringent (hi-bi) fibre enable the angle of an applied transverse load to be determined relative to the fibre by measuring the spectral shifts of the two Bragg peaks corresponding to the 'slow' and 'fast' axis polarisations [4]. Ye et al. [5] have used a polarisation maintaining fibre coupler to detect both polarisation components simultaneously to avoid ambiguity if the two Bragg peaks overlap when the loading is applied. Recently, Abe et al. [6] have used two co-located FBGs written in hi-bi fibre to measure transverse strain, longitudinal strain and temperature simultaneously.

Our interest is in measuring the effects of transverse loading applied to multicore fibre (MCF) using FBGs as the sensing element. We have previously reported the use of FBGs written in several cores of MCF to measure structural bending [7]. The axial strain imposed by bending the fibre is measurable as a differential shift in the Bragg wavelengths between pairs of cores within the fibre. A measure of the differential shift between two cores gives curvature in the plane containing the two cores - using two orthogonal cores gives a measure of bending in 2 dimensions. However, in this paper we report experiments to investigate the response of FBGs in 4-core fibre due to transverse loading. The local transverse stress at each grating will depend on the magnitude of the applied load and its orientation with respect to the core positions within the fibre. Hence the four FBG spectra should contain information from which the applied load can be derived.

\section{WRITING GRATINGS IN MULTICORE FIBRE}

The multicore fibre is fabricated from a preform in which 4 holes are drilled to accept doped glass rods that will form the cores of the fibre. The diameter of the preform and location of the holes are such that when the fibre is pulled into its final form it results in a fibre with $125 \mu \mathrm{m}$ outer diameter and a square matrix of cores with a core spacing of $50 \mu \mathrm{m}$. Each core is single mode at $1550 \mathrm{~nm}$ and distant from the other cores such that core-to-core coupling is not evident. The dopant in the core is deliberately chosen to enhance the photosensitivity of this fibre specifically for the purpose of

\footnotetext{
* email: W.N.MacPherson@ hw.ac.uk, tel +44 (0)131 451 3733, fax +44 (0)131 4513088

II Present address: Naval Research Laboratory, 4555 Overlook Avenue SW, Washington, DC 20375-5338, USA
} 
writing gratings. The fibre cross-section is illustrated in Figure 1. The circular cross-section is particularly important for fibre handling, and we have observed that higher quality cleaves can be obtained using this fibre in contrast to other (noncircular) multicore fibres that we have tested previously [7]. This has allowed us to make low-loss $(<1 \mathrm{~dB})$ splices between lengths of multicore fibre using a Vytran splicer with fibre rotation and alignment facility.

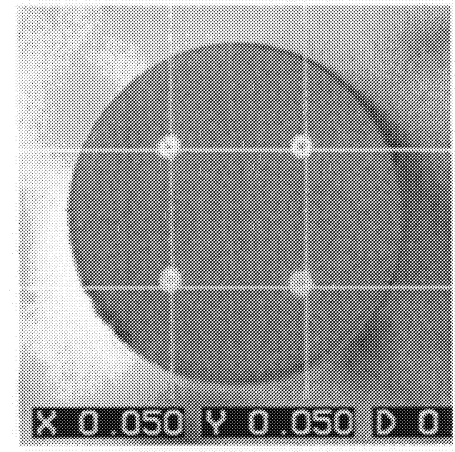

Figure 1: Cross section of the multicore fibre, illustrating the 4 cores with a spacing of $50 \mu \mathrm{m}$. The overall fibre diameter is $125 \mu \mathrm{m}$ and is therefore compatible with many traditional fibre handling techniques

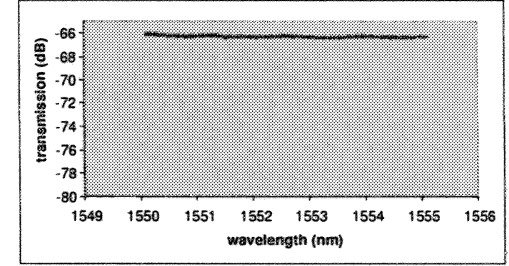

\section{Core 1}

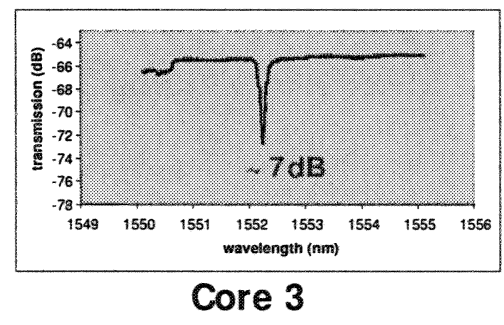

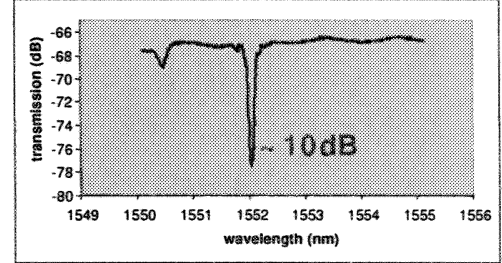

Core 2

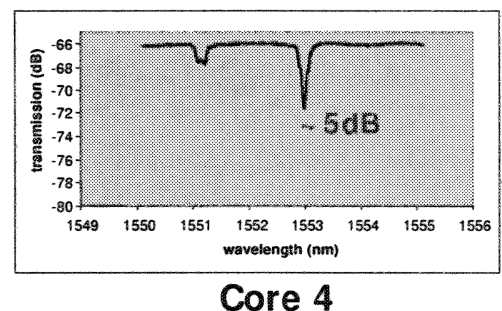

Figure 2: Spectra of FBGs in the 4-core fibre

The intrinsic photosensitivity of this multicore fibre permits UV-inscription of the FBG structures in the cores without hydrogenation of the fibre - an essential treatment for grating fabrication in standard telecom and the non-circular fourcore fibres that we have used in previous work [7]. We have produced high-efficiency FBGs in this multicore fibre using the standard phase mask scanning technique [8] and a frequency doubled Ar laser with a $95 \mathrm{~mW}$ output at $244 \mathrm{~nm}$. In the fabrication, the UV beam was scanned at a speed of $0.07 \mathrm{~mm} \mathrm{~s}^{-1}$ over a $10 \mathrm{~mm}$ length of the fibre. In order to write gratings selectively on the cores, we mounted the fibre on a rotation stage to accommodate the setting of the fibre orientation. Both single- and double-exposure methods were employed to write grating structures in one or more cores. The latter involves a second UV exposure to the fibre after it being rotated by $180^{\circ}$ following the first exposure.

The spectral characteristics of the FBGs were measured after the UV-inscription using an EDFA light source and an optical spectrum analyser. A fan-out coupling device connecting the multicore fibre to a single mode fibre was specially fabricated and used to interrogate all the multicore gratings. Figure 2 shows the transmission spectra of all four cores of a UV-inscribed multicore fibre device. It can be seen clearly from the figure that the grating structures were generated only in three cores. The FBG resonance in other gratings manufactured in this fibre has been up to $18 \mathrm{~dB}$, which is comparable to the typical FBG strength achieved in the conventional photosensitive fibres of single core. This indicates the photosensitivity of this new type of multicore fibre is sufficiently high and can be further enhanced by the hydrogenation treatment for UV-inscription of complex grating structures. We note from the figure that the central wavelengths $(1552.03 \mathrm{~nm}-1552.97 \mathrm{~nm})$ and the strengths $(5-10 \mathrm{~dB})$ of the FBGs in the three cores are slightly different. This is due to the small variations in UV intensities experienced by the three cores, which we believe can be equalised by a more controlled fabrication procedure.

\section{TRANSVERSE LOADING EXPERIMENTS}

The MCF containing FBGs in three cores was interrogated using the configuration shown in Figure 3. The MCF under test (i.e the sensing fibre) was fusion spliced onto a length of MCF that forms part of a fan-out assembly that interfaces 4 standard SMF-28 input fibres to one MCF output, such that each input addresses only one of the MCF cores. The four input fibres could be selected individually using a fibre switch (channel selector) thus enabling the reflection spectrum from each of the four cores to be interrogated sequentially using the broadband source and wavelength meter. 


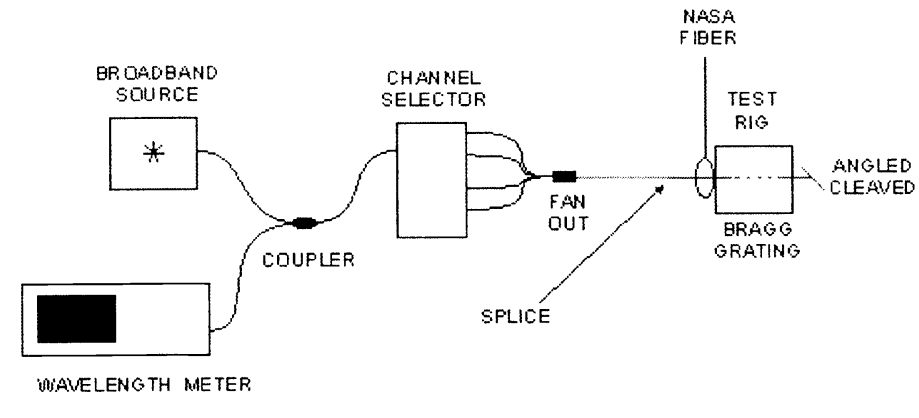

Figure 3: Optical interrogation scheme to measure FBG spectra in the MCF

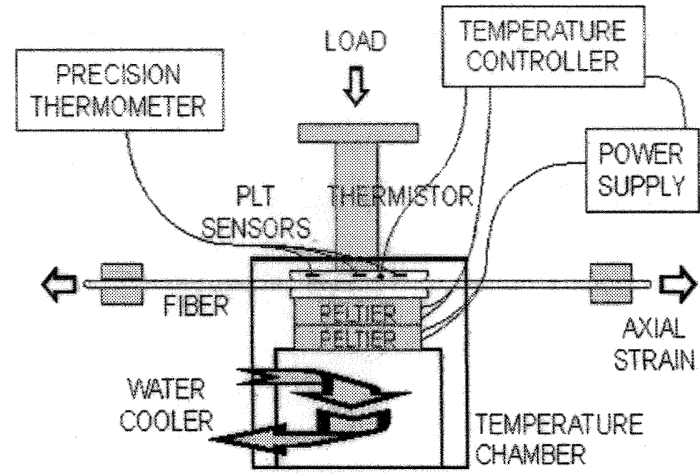

Figure 4: Experimental test chamber for control of applied transverse load, axial load, and temperature

The test rig for applying the transverse load is illustrated in Figure 4. The fibre was held between two flat polished aluminium plates such that it was parallel to a second 'dummy' fibre, which ensured that the applied load was distributed uniformly by constraining the two plates to remain parallel under loading. The load was applied by adding masses to the top aluminium plate via a system of loading plates to ensure that the load was evenly distributed.The fiber was coated with thermal oil in order to get a good thermal contact. The length of sensing fibre under load was $50 \mathrm{~mm}$. The sensing fibre could be rotated about its central axis to allow the direction of the applied transverse load to be varied relative to the fibre axis. The sensing fibre could also have a known axial load applied (via a translation stage and load cell monitor), and the temperature was controlled using water-cooled Peltier elements and suitable control units.

To aid understanding of the results it was necessary to know the orientation of the fibre prior to applying the transverse load. The position of each individual core was found by illuminating the grating with a visible laser and using a microscope to locate the position and height of the light scattered from the core. In this way it was possible to obtain the current fibre orientation. During the experiment the fibre was rotated such that load was applied directly over the third core, the fourth core and at 45 degrees between them as illustrated in Figure 5. The temperature was held at $22^{\circ} \mathrm{C}$, the axial strain was set to be $570 \mu \varepsilon$ and the load applied was $22.24 \mathrm{~kg}$.

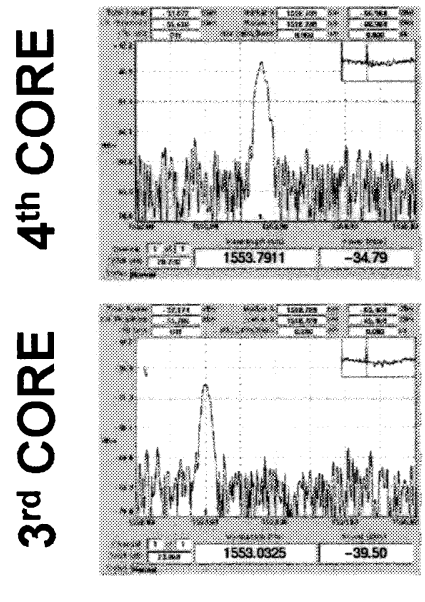

No Load

(a)

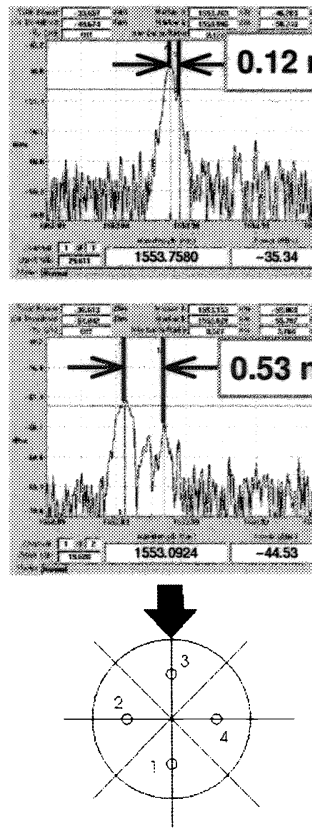

(b)
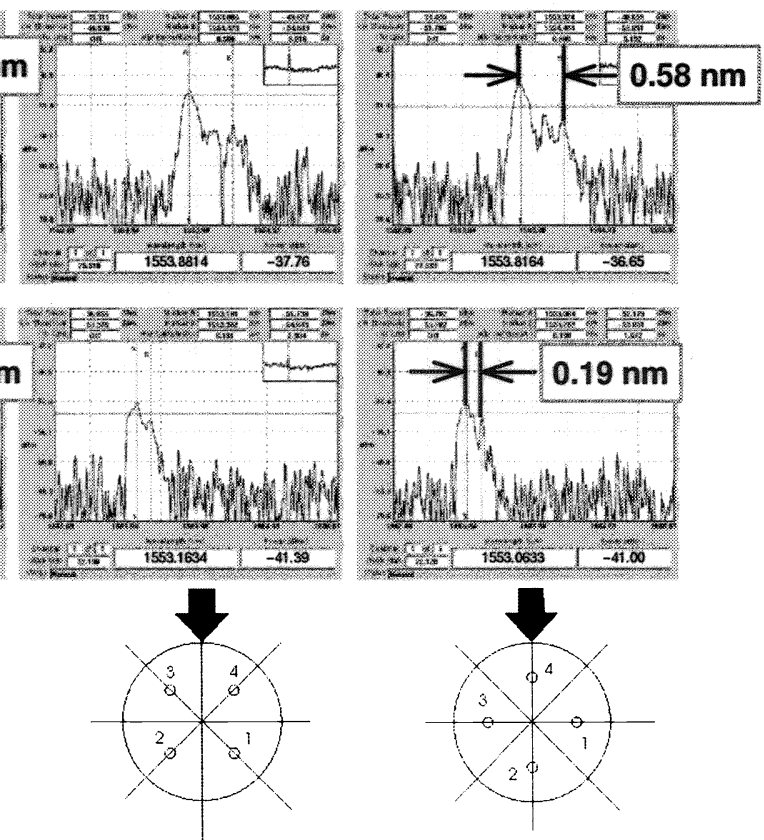

(c)

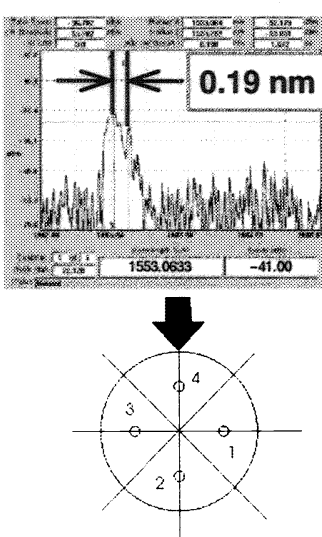

(d)

Figure 5: Experimental results from axial load testing 


\section{DISCUSSION}

Every core in the fibre is in fact bimodal as light can propagate in two nearly degenerate modes with orthogonal polarisation. Since the birefringence is a function of the stress in the core [2], we expect to observe peak splitting of the Bragg grating spectrum. In the unstressed fibre the birefringence is very small and therefore we did not observe any peak splitting. However when a transverse load is applied the stress becomes much higher in the vertical (loading) axis, especially close to the points of applied load [1], and the resulting birefringence does indeed cause a splitting of the grating reflection spectrum.

From the experimental results we observed complex FBG spectra. When two peaks are present, the second peak often contains several maxima (see figure 5) thus making it difficult to evaluate the birefringence. In spite of this it is still clear that the induced birefringence is higher in the cores located in the vertical axis close to the point of loading, which is in agreement with the theoretical analysis.

Based on the model developed by Gafsi and El-Sherif [3], we calculated the peak splitting of the Bragg grating spectrum under transverse loading. It was found that when the multicore fibre is transverse-loaded with $22.24 \mathrm{~kg}$ with the load applied adjacent to a core then the peak splitting in that core is about $0.54 \mathrm{~nm}$. This configuration is the same as figure 5 (b) for core 3 and figure 5 (d) for core 4 and is in very good agreement with our experimental results where the peak splitting is $0.53 \mathrm{~nm}$ for core 3 and $0.58 \mathrm{~nm}$ for core 4 . In this case, the calculated peak splitting ratio between the vertical cores and the horizontal cores is 3.8 which is in good agreement with experimental values of 4.4 and 3.0 for orientation in figure 5(b) and figure 5(d) respectively. The calculation also indicates that if the distance between the cores is $71.71 \mu \mathrm{m}$ rather than $50 \mu \mathrm{m}$, the peak splitting ratio between the vertical cores and the horizontal cores would increase to 21 . This means that the MCF can be designed to make the horizontally oriented cores very insensitive to the transverse loading, so that they can be easily used to simultaneously measure the longitudinal strain and temperature.

\section{CONCLUSIONS}

In this paper we report measurements of the reflection spectra of Bragg gratings inscribed into a 4-core fibre subject to transverse loading. The initial results presented here show a broadening and eventual splitting of the grating reflection peaks due to transverse strain induced birefringence. The magnitude of this effect is a function of the fibre load and orientation, and by careful inspection of the fibre we can determine the orientation, thus confirming that the magnitude of this effect relates to the strain distribution through the fibre cross section resulting from the applied load. The measured peak splitting is in good agreement with our theoretical calculation.

\section{ACKNOWLEDGEMENTS}

The authors would like to acknowledge the UK Engineering and Physical Science Research Council (EPSRC) and UK Defence Science and Technology Laboratory (dstl) for provision of funding through the Joint Grant Scheme, and W MacPherson and A Moore acknowledge the EPSRC for provision of funding via the Advanced Fellowship Programme. The authors also wish to thank Dr. G. Fleming (NASA Langley) for supply of the multicore fibre.

\section{REFERENCES}

1. P. D. Gianino, B. Bendow, Calculations of stress-induced changes in the transverse refractive-index profile of optical fibers, Applied Optics, 20 (3), $430-434,1981$

2. K. Okamoto, T. Hosaka, T. Edahiro, Stress analysis of optical fibers by a finite element method, IEEE J. Quantum Electronics, QE17 (10), $2123-2129,1981$

3. R. Gafsi, M. A. El-Sherif, Analysis of induced-birefringence effects on fiber Bragg gratings, Optical Fiber Tech. 6, 299-323, 2000

4. C. M. Lawrence, D. V. Nelson, E. Udd, T. Bennett, A fiber optic sensor for transverse strain measurement, Experimental

Mechanics, 39 (3), $202-209,1999$

5. C.C. Ye, S.E. Staines, S.W. James, R.P. Tatam, A polarization-maintaining fibre Bragg grating interrogation system for multi-axis strain sensing, Measurement Science \& Technology, 13, 1446 - 1449, 2002

6. I. Abe, O. Frazao, R.N. Nogueira, H.J. Kalinowski, J.L. Pinto, J.L. Santos, Three-parameter simultaneous measurement using superimposed Bragg gratings in high-birefringence optical fibers, $16^{\text {th }}$ Int. Conf. on Optical Fiber Sensors, Technical Digest, Inst. of Electronics, Information and Communication Engineers 2003, ISBN 4-89114-036-4, 96 - 99, 2003

7. G. M. H. Flockhart, W. N. MacPherson, J. S. Barton, J. D. C. Jones, L. Zhang, I. Bennion , 'Two-axis bend measurement with Bragg gratings in multicore optical fiber', Optics Letters, 28 (6), 387-389, 2003

8. K. O. Hill, B. Malo, F. Bilodeau, D. C. Johnson, and J. Albert, "Bragg gratings fabricated in monomode photosensitive optical fibre by UV exposure through a phase mask," Appl. Phys. Lett., 62, pp. 1035-1037, 1993 